\title{
ON CERTAIN FAMILIES OF ORBITS WITH ARBITRARY MASSES IN THE PROBLEM OF THREE BODIES (SECOND PAPER)*
}

BY

\section{F. H. MURRAY}

It is the object of this paper to obtain theorems concerning the stability of the straight line solutions, and equilateral triangle solutions, respectively, in the problem of three bodies by means of the theorems and calculations of two preceding papers. $\dagger$ It is shown that the generalized theorems of Bohl can be applied to a neighborhood of the straight line solutions, with arbitrary masses, and to a neighborhood of the equilateral triangle solutions, if the masses are such that the characteristic exponents of the generating orbit are not all pure imaginaries. The mutual distances of the three masses are assumed constant on the generating orbit, in both cases.

\section{The equations of Motion}

From the classical reduction of the equations of motion of the problem of three bodies these are obtained in the form

$$
\begin{aligned}
& \frac{d \bar{x}_{i}}{d t}=\frac{\partial F}{\partial \bar{y}_{i}}, \frac{d \bar{y}_{i}}{d t}=-\frac{\partial F}{\partial \bar{x}_{i}} \quad(i=1,2, \ldots, 6), \\
& F=\frac{h}{2} \sum_{i=1}^{3} \bar{y}_{i}^{2}+\frac{g}{2} \sum_{i=4}^{6} \bar{y}_{i}^{2}-U, \quad U=\frac{m_{1} m_{2}}{r_{0}}+\frac{m_{2} m_{0}}{r_{1}}+\frac{m_{0} m_{1}}{r_{2}}, \\
& r_{0}^{2}=\left(\bar{x}_{4}-\mu \bar{x}_{1}\right)^{2}+\left(\bar{x}_{5}-\mu \bar{x}_{2}\right)^{2}+\left(\bar{x}_{6}-\mu \bar{x}_{3}\right)^{2}, \\
& r_{1}^{2}=\left(\bar{x}_{4}+\lambda \bar{x}_{1}\right)^{2}+\left(\bar{x}_{5}+\lambda \bar{x}_{2}\right)^{2}+\left(\bar{x}_{8}+\lambda \bar{x}_{3}\right)^{2}, \\
& r_{2}^{2}=\bar{x}_{1}^{2}+\bar{x}_{2}^{2}+\bar{x}_{3}^{2}, \quad \mu=\frac{m_{0}}{m_{0}+m_{1}}, \\
& \lambda=\frac{m_{1}}{m_{0}+m_{1}}, \quad h=\frac{m_{0}+m_{1}}{m_{0} m_{1}} . \\
& g=\frac{m_{0}+m_{1}+m_{2}}{m_{2}\left(m_{0}+m_{1}\right)}, \quad h \quad
\end{aligned}
$$

- Presented to the Society, December 30, 1924; received by the editors in April, 1925.

$\dagger$ (B) Generalization of certain theorems of Bohl, American Journal of Mathematics, vol. 47 (1925); (A) On certain families of orbits with arbitrary masses in the problem of three bodies, these Transactions, vol. 28 (1926), pp. 74-108. 
$f$ the transformation to new coördinates is made,

$$
\begin{aligned}
& \bar{x}_{1}=\rho_{1} \cos \varphi_{1} \quad \bar{x}_{1}=\rho_{2} \cos \varphi_{2}, \quad \bar{m}_{1}=\frac{1}{h}, \\
& \bar{x}_{2}=\rho_{1} \sin \varphi_{1} \quad \bar{x}_{5}=\rho_{2} \sin \varphi_{2}, \quad \bar{m}_{2}=\frac{1}{g},
\end{aligned}
$$

equations (1) become

where

$$
\begin{array}{lll}
\frac{d \rho_{k}}{d t}=\frac{\partial F}{\partial R_{k}}, & \frac{d R_{k}}{d t}=-\frac{\partial F}{\partial \rho_{k}}, & \\
\frac{d \varphi_{k}}{d t}=\frac{\partial F}{\partial G_{k}}, & \frac{d G_{k}}{d t}=-\frac{\partial F}{\partial \varphi_{k}} & (k=1,2), \\
\frac{d \bar{x}_{i}}{d t}=\frac{\partial F}{\partial \bar{y}_{i}}, & \frac{d \bar{y}_{i}}{d t}=-\frac{\partial F}{\partial \bar{x}_{i}} & (i=3,6),
\end{array}
$$

$$
\begin{aligned}
& F=\frac{1}{2 \bar{m}_{1}}\left(R_{1}^{2}+\frac{G_{1}^{2}}{\rho_{1}^{2}}+\bar{y}_{3}^{2}\right)+\frac{1}{2 \bar{m}_{2}}\left(R_{2}^{2}+\frac{G_{2}^{2}}{\rho_{2}^{2}}+\bar{y}_{8}^{2}\right)-U, \\
& r_{0}^{2}=\rho_{2}^{2}+\mu^{2} \rho_{1}^{2}-2 \mu \rho_{1} \rho_{2} \cos \left(\varphi_{2}-\varphi_{1}\right)+\left(\bar{x}_{6}-\mu \bar{x}_{3}\right)^{2}, \\
& r_{1}^{2}=\rho_{2}^{2}+\lambda^{2} \rho_{1}^{2}+2 \lambda \rho_{1} \rho_{2} \cos \left(\varphi_{2}-\varphi_{1}\right)+\left(\bar{x}_{6}+\lambda \bar{x}_{8}\right)^{2} \\
& r_{2}^{2}=\rho_{1}^{2}+\bar{x}_{3}^{2} .
\end{aligned}
$$

On account of the area integral

$$
\left(\bar{x}_{1} \bar{y}_{2}-\bar{x}_{2} \bar{y}_{1}\right)+\left(\bar{x}_{4} \bar{y}_{5}-\bar{x}_{6} \bar{y}_{4}\right)=\text { const. , }
$$

we have

If the transformation

$$
G_{1}+G_{2}=C
$$

$$
\begin{aligned}
\varphi_{1}^{\prime} & =\varphi_{1}, & & \varphi=\varphi_{2}-\varphi_{1}, \\
C & =G_{1}+G_{2}, & & G=G_{2}
\end{aligned}
$$

is made, the canonical form of the equations is retained, and the new equations are

(6)

$$
\begin{array}{ll}
\frac{d \varphi}{d t}=\frac{\partial F}{\partial G}, \frac{d G}{d t}=-\frac{\partial F}{\partial \varphi}, \frac{d \varphi_{1}^{\prime}}{d t}=\frac{\partial F}{\partial C}, \frac{d C}{d t}=0, & (i=1,2), \\
\frac{d \rho_{i}}{d t}=\frac{\partial F}{\partial R_{i}}, \frac{d R_{i}}{d t}=-\frac{\partial F}{\partial \rho_{i}} & (i=3,6), \\
\frac{d \bar{x}_{i}}{d t}=\frac{\partial F}{\partial \bar{y}_{i}}, \frac{d \bar{y}_{i}}{d t}=-\frac{\partial F}{\partial \bar{x}_{i}} & (i=1)
\end{array}
$$


where

$$
F=\frac{1}{2 \bar{m}_{1}}\left[R_{1}^{2}+\frac{(C-G)^{2}}{\rho_{1}^{2}}+\bar{y}_{3}^{2}\right]+\frac{1}{2 \bar{m}_{2}}\left[R_{2}^{2}+\frac{G^{2}}{\rho_{2}^{2}}+\bar{y}_{6}^{2}\right]-U .
$$

We have also

$$
\begin{array}{ll}
r_{0}^{2}=\bar{r}_{0}^{2}+\left(\bar{x}_{8}-\mu \bar{x}_{3}\right)^{2}, & \bar{r}_{0}^{2}=\rho_{2}^{2}+\mu^{2} \rho_{1}^{2}-2 \mu \rho_{1} \rho_{2} \cos \varphi, \\
r_{0}^{2}=\bar{r}_{1}^{2}+\left(\bar{x}_{8}+\lambda \bar{x}_{3}\right)^{2}, & \bar{r}_{1}^{2}=\rho_{2}^{2}+\lambda^{2} \rho_{1}^{2}+2 \lambda \rho_{1} \rho_{2} \cos \varphi, \\
r_{2}^{2}=\rho_{1}^{2}+\bar{x}_{3}^{2} . &
\end{array}
$$

For later computations it will be necessary to expand the function $F$ in powers of $\bar{x}_{3}, \bar{x}_{6}$. We have

(8)

$$
\begin{aligned}
\frac{1}{r_{0}} & =\frac{1}{\bar{r}_{0}}\left[1+\left(\frac{\bar{x}_{6}-\mu \bar{x}_{3}}{\bar{r}_{0}}\right)^{2}\right]^{-1} \\
& =\frac{1}{\bar{r}_{0}}\left[1-\frac{1}{2}\left(\frac{\bar{x}_{6}-\mu \bar{x}_{3}}{\bar{r}_{0}}\right)^{2}+\frac{1 \cdot 3}{2 \cdot 4}\left(\frac{\bar{x}_{8}-\mu \bar{x}_{3}}{\bar{r}_{0}}\right)^{4}+\cdot \cdot\right],
\end{aligned}
$$

$$
\begin{aligned}
\frac{1}{r_{1}} & =\frac{1}{\bar{r}_{1}}\left[1+\left(\frac{\bar{x}_{6}+\lambda \bar{x}_{3}}{\bar{r}_{1}}\right)^{2}\right]^{-1} \\
& =\frac{1}{\bar{r}_{1}}\left[1-\frac{1}{2}\left(\frac{\bar{x}_{8}+\lambda \bar{x}_{3}}{\bar{r}_{1}}\right)^{2}+\frac{1 \cdot 3}{2 \cdot 4}\left(\frac{\bar{x}_{6}+\lambda \bar{x}_{3}}{\bar{r}_{1}}\right)^{4}+\cdot \cdot\right],
\end{aligned}
$$$$
\frac{1}{r_{2}}=\frac{1}{\rho_{1}}\left[1+\left(\frac{\bar{x}_{3}}{\rho_{1}}\right)^{2}\right]^{-\frac{1}{1}}
$$$$
=\frac{1}{\rho_{1}}\left[1-\frac{1}{2}\left(\frac{\bar{x}_{3}}{\rho_{1}}\right)^{2}+\frac{1 \cdot 3}{2 \cdot 4}\left(\frac{\bar{x}_{3}}{\rho_{1}}\right)^{4}+. \cdot\right] \text {. }
$$

Consequently,

$$
\begin{aligned}
U=\frac{m_{1} m_{2}}{\bar{r}_{0}}+\frac{m_{2} m_{0}}{\bar{r}_{1}}+\frac{m_{0} m_{1}}{\bar{r}_{2}} & -\frac{1}{2}\left[m_{1} m_{2} \frac{\left(\bar{x}_{6}-\mu \bar{x}_{3}\right)^{2}}{\bar{r}_{0}^{3}}\right. \\
& \left.+m_{2} m_{0} \frac{\left(\bar{x}_{6}+\lambda \bar{x}_{3}\right)^{2}}{\bar{r}_{1}^{0}}+m_{0} m_{1} \frac{\bar{x}_{2}^{2}}{\rho_{1}^{3}}\right]+\bar{U},
\end{aligned}
$$

and

$$
\begin{aligned}
& F=F_{1}+F_{2}+F_{3} \\
& F_{1}=\frac{1}{2 \bar{m}_{1}}\left[R_{1}^{2}+\frac{(C-G)^{2}}{\rho_{1}^{2}}\right]+\frac{1}{2 \bar{m}_{2}}\left[R_{2}^{2}+\frac{G^{2}}{\rho_{2}^{2}}\right]-\frac{m_{1} m_{2}}{\bar{r}_{0}}-\frac{m_{2} m_{0}}{\bar{r}_{1}}-\frac{m_{0} m_{1}}{\bar{r}_{2}} \\
& F_{2}=\frac{\bar{y}_{3}^{2}}{2 \bar{m}_{1}}+\frac{\bar{y}_{6}^{2}}{2 \bar{m}_{2}}+\frac{m_{1} m_{2}}{2} \frac{\left(\bar{x}_{6}-\mu \bar{x}_{3}\right)^{2}}{\bar{r}_{0}^{3}}+\frac{m_{2} m_{0}}{2} \frac{\left(\bar{x}_{8}+\lambda \bar{x}_{3}\right)^{2}}{\bar{r}_{1}^{3}}+\frac{m_{0} m_{1}}{2} \frac{\bar{x}_{3}^{2}}{\rho_{1}^{2}}
\end{aligned}
$$


and $F_{3}$ contains only powers of $\bar{x}_{3}, \bar{x}_{6}$, not lower than the third.

Since the invariant relations

$$
\bar{x}_{3}=\bar{x}_{6}=\bar{y}_{3}=\bar{y}_{6}=0
$$

define a movement in the plane, these may be assumed satisfied in the equations above if it is desired to study the orbits in this plane.

2. ORBITS IN THE NEIGHBORHOOD OF THE STRAIGHT IINE SOLUTIONS

In (A), $\S 8$, it was shown that if the equations $(10, \S 1)$, are satisfied, linear combinatiuns of the remaining variables can be so chosen that the equations of motion retain the canonical form, while the straight line solutions are characterized by the invariant relations*

$$
u_{1}=u_{2}=v_{1}=v_{2}=0 \text {. }
$$

When these relations are satisfied, the function $F$ becomes, if $\rho_{1}{ }^{\prime \prime}=\rho_{1}$, and

$$
m^{\prime}=\bar{m}_{1}+\bar{m}_{2}(\mu+\nu)^{2}, \quad M^{\prime} m^{\prime}=m_{0} m_{1}+\frac{m_{0} m_{2}}{1+\nu}+\frac{m_{1} m_{2}}{\nu},
$$

(2) $\quad F^{0}=\frac{1}{2 m^{\prime}}\left[R_{1}{ }^{\prime 2}+\frac{C^{2}}{\rho_{1}{ }^{\prime 2}}\right]-\frac{M^{\prime} m^{\prime}}{\rho_{1}^{\prime \prime}}$.

Equations (1) being satisfied, the remaining equations

$$
\frac{d \rho_{1}{ }^{\prime \prime}}{d t}=\frac{1}{m^{\prime}} R_{1}^{\prime \prime}, \quad \frac{d R_{1}{ }^{\prime \prime}}{d t}=-\frac{\partial F^{0}}{\partial \rho_{1}^{\prime \prime}}
$$

are satisfied by $R_{1}^{\prime \prime}=0, \rho_{1}^{\prime \prime}=\rho_{1}$ if

$$
\frac{\partial F^{0}}{\partial \rho_{1}{ }^{\prime \prime}}=-\frac{C^{2}}{m^{\prime} \rho_{1}{ }^{\prime \prime 3}}+\frac{M^{\prime} m^{\prime}}{\rho_{1}{ }^{\prime \prime 2}}=0, \quad \rho_{1}^{\prime \prime}=\rho_{1}=\frac{C^{2}}{M^{\prime} m^{\prime 2}} \text {. }
$$

Suppose $\rho_{1}^{\prime \prime}=\rho_{1}+\xi, R_{1}^{\prime \prime}=\eta$; the function $F^{0}$ becomes

$$
F^{0}=\frac{\eta^{2}}{2 m^{\prime}}+\frac{M^{\prime} m^{\prime}}{2 \rho_{1}^{3}} \xi^{2}+F_{3}^{0}
$$

where $F_{3}^{0}$ contains only powers of $\xi$ higher than the second. The relations $\xi=\eta=0$ characterize the orbits for which the mutual distances $r_{0}, r_{1}, r_{2}$ are constant. If $\bar{F}_{1}=F_{1}-F^{0}$, we obtain

$$
F=\frac{\eta^{2}}{2 m^{\prime}}+\frac{M^{\prime} m^{\prime}}{2 p_{1}^{3}} \xi^{2}+\bar{F}_{1}+F_{2}+F_{3}+F_{3}^{0} .
$$

- See (A), \$\$1, 2; Poincare, Les Methodes Nouvelles, vol. 1, \$19. 
In $\S 9,(\mathrm{~A})$, it was seen that if $\bar{F}_{1}$ is expanded as a power series in the variables $u_{1}, u_{2}, v_{1}, v_{2}$, only terms of the second and higher orders occur. It is now a simple matter to write down the equations of variation corresponding to equations (6), $\$ 1$. These become

(a) $\frac{d \xi}{d t}=\frac{\eta}{m^{\prime}}, \frac{d \eta}{d t}=-\frac{M^{\prime} m^{\prime}}{\rho_{1}^{3}} \xi ;$

$$
\begin{array}{ll}
\frac{d \mathfrak{a}_{1}}{d t}= & A_{22} \bar{v}_{1}, \\
\frac{d a_{2}}{d t}=B_{12} a_{1} & +B_{22} \bar{v}_{2},
\end{array}
$$

(5)

(b)

$$
\frac{d \bar{v}_{1}}{d t}=-A_{11} a_{1} \quad-B_{12} \bar{v}_{2},
$$$$
\frac{d \bar{v}_{2}}{d t}=\quad-B_{11} \mathfrak{a}_{2} ;
$$$$
\frac{d \xi_{3}}{d t}=\frac{\eta_{3}}{\bar{m}_{1}},
$$$$
\frac{d \xi_{0}}{d t}=\frac{\eta_{6}}{\bar{m}_{2}},
$$

(c)

$$
\begin{aligned}
& \frac{d \eta_{3}}{d t}=-\left[\frac{\lambda^{2} m_{2} m_{0}}{\bar{r}_{1}^{3}}+\frac{\mu^{2} m_{1} m_{2}}{\bar{r}_{0}^{3}}+\frac{m_{0} m_{1}}{\rho_{1}^{3}}\right] \xi_{3}+\left[\frac{m_{1} m_{2} \mu}{\vec{r}_{0}^{3}}-\frac{m_{2} m_{0} \lambda}{\vec{r}_{1}^{3}}\right] \xi_{0}, \\
& \frac{d \eta_{6}}{d t}=\left[\frac{\mu m_{1} m_{2}}{\bar{r}_{0}^{3}}-\frac{\lambda m_{2} m_{0}}{\vec{r}_{1}^{3}}\right] \xi_{3}-\left[\frac{m_{1} m_{2}}{\vec{r}_{0}^{3}}+\frac{m_{2} m_{0}}{\vec{r}_{1}^{3}}\right] \xi_{0} .
\end{aligned}
$$

If equations $(5, c)$ are written in the form

(6)

$$
\begin{aligned}
& \frac{d \xi_{3}}{d t}=\frac{\eta_{3}}{\bar{m}_{1}}, \frac{d \xi_{6}}{d t}=\frac{\eta_{6}}{\bar{m}_{2}}, \\
& \frac{d \eta_{3}}{d t}=-C_{11} \xi_{3}+C_{12} \xi_{6}, \\
& \frac{d \eta_{6}}{d t}=C_{12} \xi_{3}-C_{22} \xi_{0},
\end{aligned}
$$

it is easily seen that the characteristic exponents $s$ must satisfy

$$
\bar{m}_{1} \bar{m}_{2} s^{4}+\left[C_{11} \bar{m}_{2}+C_{22} \bar{m}_{1}\right] s^{2}+C_{11} C_{22}-C_{12}^{2}=0,
$$


and consequently are all pure imaginaries. It is also evident that the exponents corresponding to $(5, a)$ are pure imaginaries; from the calculations of $(\mathrm{A}), \S 9$, it has been seen that two exponents are pure imaginaries, while the remaining two are real, corresponding to the equations $(5, b)$.

In order to apply the generalized theorems of Bohl it only remains to calculate the numbers $\epsilon_{i}$ corresponding to these eight pure imaginary exponents; from (4) it is seen that the terms of the second order in $\xi, \eta$, $\bar{x}_{3}, \bar{x}_{6}, \bar{y}_{3}, \bar{y}_{6}$, occurring in the expansion of $F$ form a positive definite form in these variables, and since the variables employed in (B), \$2, are obtained by a real linear transformation, the new corresponding form must also be positive definite (as is easily verified directly). It only remains to calculate the values of $\epsilon_{i}$ corresponding to $(5, b)$. The corresponding exponents satisfy

$$
s^{4}+\left[A_{11} A_{22}+B_{11} B_{22}\right] s^{2}+B_{11} A_{22}\left[A_{11} B_{22}-B_{12}^{2}\right]=0,
$$

and if a solution of $(b)$ is assumed in the form

$$
a_{1}=\xi_{1} e^{a t}, \quad a_{2}=\xi_{2} e^{e t}, \quad \bar{v}_{1}=\eta_{1} e^{a t}, \quad \bar{v}_{2}=\eta_{2} e^{e t},
$$

we obtain the relations

$$
\begin{aligned}
-s \cdot \xi_{1}+0 \cdot \xi_{2}+A_{22} \eta_{1}+0 \cdot \eta_{2} & =0, \\
B_{12} \xi_{1}-s \cdot \xi_{2}+0 \cdot \eta_{1}+B_{22} \eta_{2} & =0, \\
-A_{11} \xi_{1}+0 \cdot \xi_{2}-s \cdot \eta_{1}-B_{12} \eta_{2} & =0, \\
0 \cdot \xi_{1}-B_{11} \xi_{2}+0 \cdot \eta_{1}-s \cdot \eta_{2} & =0 .
\end{aligned}
$$

If $s$ satisfies (8), and is of the form $p \sqrt{-1}, p>0$, the third line can be omitted; if the values of $\xi_{1}, \xi_{2}, \eta_{1}, \eta_{2}$ are chosen equal to the corresponding threerowed determinants remaining,

$$
\left(\xi_{1} \bar{\eta}_{1}-\eta_{1} \bar{\xi}_{1}\right)+\left(\xi_{2} \bar{\eta}_{2}-\eta_{2} \bar{\xi}_{2}\right)=-2 A_{22} s\left\{\left(s^{2}+B_{11} V_{22}\right)^{2}+A_{28} B_{11} B_{12}^{2}\right\},
$$

the dashed constants being the conjugate imaginaries of the corresponding ones with the same subscripts.

Since $A_{22}$ and $B_{11}$ are positive (see (A), $\S$ ), it follows that if each of the constants $\xi_{i}, \bar{\xi}_{i}, \eta_{i}, \bar{\eta}_{i}(i=1,2)$ is divided by the real constant

$$
\sqrt{-2 A_{28} 2^{2}\left[\left(s^{2}+B_{11} B_{2}\right)^{2}+A_{28} B_{11} B_{12}^{2}\right]}
$$

the resulting constants will be of the form required for the application of the theorems of $(B)$, and $\epsilon_{i}=1 / s_{i}$. Hence the terms of the second order in the expansion of $F$ corresponding to the eight imaginary exponents will be 
a positive definite form, and the generalized theorems of Bohl can be applied.* Suppose new variables $x_{1}, x_{2}, x_{3}, x_{4}, y_{1}, y_{2}, y_{3}, y_{4}, u_{1}^{\prime}, v_{1}^{\prime}$ introduced as in (B), §2, Part I. The function $F$ is analytic in these variables, consequently a constant $d$ can be chosen so small that from (B), II, $\$ 4$, we have the following theorems:

1. To every set of values of the region $(R)$,

$$
\sum_{i=1}^{4}\left(x_{i}^{2}+y_{i}^{2}\right) \leqq 2 d^{2} / 9, \quad\left|v_{1}^{\prime}\right| \leqq d / 2,
$$

can be adjoined a value $u_{1}{ }^{\prime},\left|u_{1}{ }^{\prime}\right| \leqq d / 2$ such that the set of values $\left(x_{i}, y_{i}, v_{1}{ }^{\prime}\right)$, $u_{1}{ }^{\prime}$ are the initial values of a trajectory which remains in the region

$$
\sum_{i=1}^{4}\left(x_{i}^{2}+y_{i}^{2}\right) \leqq d^{2}, \quad\left|u_{1}^{\prime}\right| \leqq d / 2, \quad\left|0_{1}^{\prime}\right| \leqq d / 2
$$

for $t>t_{0}$, and this value is uniquely determined.

2. To every set of values $\left(x_{i}, y_{i}, u_{1}^{\prime}\right)$ of the region $\left(R^{\prime}\right)$,

$$
\sum_{i=1}^{4}\left(x_{i}^{2}+y_{i}^{2}\right) \leqq 2 d^{2} / 9, \quad\left|u_{1}^{\prime}\right| \leqq d / 2,
$$

corresponds a value $v_{1}^{\prime},\left|v_{1}{ }^{\prime}\right| \leqq d / 2$, such that the trajectory with this set of initial values at $t=t_{0}$ lies in the region $(C)$ for $t<t_{0}$, and this value is uniquely determined.

3. To every set of values of the region

$$
\sum_{i=1}^{4}\left(x_{i}^{2}+y_{i}^{2}\right) \leqq 2 d^{2} / 9
$$

corresponds $a$ set of values of the region $\left|u_{1}{ }^{\prime}\right| \leqq d / 2,\left|v_{1}{ }^{\prime}\right| \leqq d / 2$, such that the trajectory with these initial values at $t=t_{0}$ remains in the region $(C)$ for all values of $t$; and the values of $u_{1}{ }^{\prime}, v_{1}{ }^{\prime}$ are uniquely determined.

3. ORBITS IN THE NEIGHBORHOOD OF THE EQUILATERAL TRIANGLE SOLUTIONS

From the calculations of (A), $\S 8$, it is seen that when the invariant relations characterizing an equilateral triangle solution are satisfied, the function $F_{1}$ of $(9), \S 1$, becomes

$$
F_{0}=\frac{1}{2 \bar{m}}\left[R_{1}^{\prime \prime 2}+\frac{C^{2}}{\rho_{1}^{\prime \prime 2}}\right]-\frac{M \bar{m}}{\rho_{1}^{\prime \prime}},
$$

$$
\bar{m}=\bar{m}_{1}+\gamma^{2} \bar{m}_{2}=\frac{m_{0} m_{1}+m_{1} m_{2}+m_{2} m_{0}}{M}, \quad M=m_{0}+m_{1}+m_{2} .
$$

\footnotetext{
* (B), Part II, \$2.
} 
If $\rho$ is a constant value of $\rho_{1}{ }^{\prime \prime}$ corresponding to a solution in which the mutual distances $r_{0}, r_{1}, r_{2}$ are constant, then, as in $\$ 2$,

$$
\rho=C^{2} / M \bar{m}^{2},
$$

and if $\rho_{1}{ }^{\prime \prime}=\rho+\xi, R_{1}{ }^{\prime \prime}=\eta$, the function $F_{0}$ becomes

$$
F_{0}=\frac{\eta^{2}}{2 \bar{m}}+\frac{M \bar{m}}{2 \rho^{3}} \xi^{2}+\bar{F}_{3}^{0},
$$

where $\bar{F}_{3}^{0}$ contains only powers of $\xi$ greater than the second. If $F_{1}{ }^{\prime}=F_{1}-F_{0}$, then

$$
F=\frac{\eta^{2}}{2 \bar{m}}+\frac{M \bar{m}}{2 \rho^{3}} \xi^{2}+F_{1}^{\prime}+F_{2}+F_{3}+\bar{F}_{3}^{0} .
$$

In $\$ 10$, (A), it was found that if $F_{1}{ }^{\prime}$ is expanded as a power series in $u_{1}, u_{2}, v_{1}, v_{2}$ only terms of the second and higher orders occur. The equations of variation corresponding to $(5, b, \S 2)$ have a different form, but the remaining equations of variation are formally similar to the corresponding ones of $(5, \S 2)$. In $\S 10,(\mathrm{~A})$, it has been seen that the characteristic exponents are either all pure imaginaries, or they contain a group of two pairs of conjugate exponents, according as a certain function of the masses is positive or negative. If the four complex exponents occur, the conditions necessary for the transformation of (B) are seen to be satisfied; it is not necessary to calculate the numbers $\epsilon_{i}$, since the corresponding terms in $F$ (from $F_{0}+$ $F_{2}$ ) are known to form a positive definite form ; and the linear transformations to the variables used in the theorems of (B) are real. As the function $F$ is analytic within the region considered here, the generalized theorems of Bohl can be applied. Suppose new variables $x_{1}, x_{2}, x_{3}, y_{1}, y_{2}, y_{3}, \bar{u}_{1}, \bar{u}_{2}, \bar{v}_{1}$, $\bar{v}_{2}$ introduced as in (B), §2, Part I. The constant $d$ can then be chosen so small that the following theorems hold:

1. To every set of values of the region $\left(R_{1}\right)$,

$$
\sum_{i=1}^{3}\left(x_{i}^{2}+y_{i}^{2}\right) \leqq 2 d^{2} / 9, \quad \bar{v}_{1}^{2}+\bar{v}_{2}^{2} \leqq d^{2} / 4,
$$

can be adjoined a set of values of the region $\bar{u}_{1}^{2}+\bar{u}_{2}^{2} \leqq d^{2} / 4$, such that the set of values $\left(x_{i}, y_{i}, \bar{v}_{r}\right),\left(\bar{u}_{r}\right)$ form the initial values at $t=t_{0}$ of a trajectory which remains in the region

$$
\sum_{i=1}^{3}\left(x_{i}^{2}+y_{i}^{2}\right) \leqq d^{2}, \quad a_{1}^{2}+u_{2}^{2} \leqq d^{2} / 4, \quad v_{1}^{2}+v_{2}^{2} \leqq d^{2} / 4
$$

for $t>t_{0}$, and this set of values is uniquely determined. 
2. To every set of values $\left(x_{i}, y_{i}, \bar{u}_{r}\right)$ of the region $\left(R_{1}{ }^{\prime}\right)$,

$$
\sum_{i=1}^{3}\left(x_{i}^{2}+y_{i}^{2}\right) \leqq 2 d^{2} / 9, \quad a_{1}^{2}+a_{2}^{2} \leqq d^{2} / 4,
$$

can be adjoined a set of values of the region $\bar{v}_{1}^{2}+\bar{v}_{2}^{2} \leqq d^{2} / 4$, such that the set of values $\left(x_{i}, y_{i}, \bar{u}_{r}\right),\left(\bar{v}_{r}\right)$ form the initial values at $t=t_{0}$ of a trajectory which remains in $\left(C^{\prime}\right)$ for $t<t_{0}$, and this set of values is uniquely determined.

3. To every set of values of we region

$$
\sum_{i=1}^{3}\left(x_{i}^{2}+y_{i}^{2}\right) \leqq 2 d^{2} / 9
$$

corresponds a set of values of the region

$$
\bar{u}_{1}^{2}+\bar{u}_{2}^{2} \leqq d^{2} / 4, \quad \bar{v}_{1}^{2}+\bar{v}_{2}^{2} \leqq d^{2} / 4
$$

such that the trajectory with these initial values at $t=t_{0}$ remains in the region $\left(C^{\prime}\right)$ for all values of $t$; and the set of values $\left(\bar{u}_{r}\right),\left(\bar{v}_{r}\right)$ is.uniquely determined.

If we put $\rho=a$ in the expression above for $F$, it has been found that the characteristic equation corresponding to the analogue of equations $(5, b, \S 2)$ can be written*

$$
s^{4}+\frac{M}{a^{3}} s^{2}+\frac{27}{4 a^{6}}\left[m_{0} m_{1}+m_{1} m_{2}+m_{2} m_{0}\right]=0,
$$

or if $n=\sqrt{M / a^{3}}$ and $s=a n$

from which

$$
M^{2} a^{4}+M^{2} a^{2}+\frac{27}{4} M \bar{m}=0,
$$

$$
a= \pm \sqrt{-\frac{1}{2} \pm \frac{1}{2} \sqrt{1-27 \bar{m} / M}}
$$

In order for the theorems above to be valid, we must have

$$
M-27 \bar{m}<0, \text { or } M^{2}<27\left(m_{0} m_{1}+m_{1} m_{2}+m_{2} m_{0}\right) \text {. }
$$

The inequality is not satisfied, in particular, when two masses are small in comparison with the remaining one.

It is easily seen that when the last inequality is not satisfied, the roots being all distinct, the characteristic exponents are all pure imaginaries, but the quadratic form obtained in the expansion of $F$, according to the

*(A), §10. 
methods of (B), is indefinite, containing eight positive and two negative terms. Hence the generalized theorems of Bohl cannot be applied here.

\section{ConcLusion}

If the new variables which appear in the theorems of the preceding paragraphs are chosen according to the method of (B), there will be four of the variables corresponding to the pure imaginary exponents which are linear combinations of the variables $\bar{x}_{3}, \bar{x}_{6}, \bar{y}_{3}, \bar{y}_{6}$; if these new variables are equated to zero, the variables $\bar{x}_{3}, \bar{x}_{6}, \bar{y}_{3}, \bar{y}_{6}$ will themselves be zero, and the motion will take place in the plane corresponding. Hence the theorems corresponding to those of the two preceding paragraphs, relating to the motion in the plane, can be obtained by supposing four of the variables which correspond to the pure imaginary exponents, for instance $x_{1}, x_{2}, y_{1}, y_{2}$, constantly zero.

It follows immediately that the only plane orbits which remain in the neighborhood of the given equilateral triangle orbit $(M<27 \bar{m})$ for all values of the time are equilateral triangle orbits; for if the eight equations characterizing an equilateral triangle orbit in the plane $\bar{x}_{3}=\bar{x}_{6}=\bar{y}_{3}=\bar{y}_{6}=0$ are satisfied, there remain two initial values for the coördinates which can be chosen arbitrarily in a suitably restricted region. But in the plane there are just two initial values, which can be chosen arbitrarily for an orbit remaining in the given neighborhood for all values of $t$, the orbit being then uniquely determined; since these initial values are linear combinations of the initial values of the original coördinates, the two orbits must coincide.

DaLHoUste UNIVERSTTY, HaLIPAX, Nova Scotia 